\title{
Repercusión científica de las ayudas de la Delegación del Gobierno para el Plan Nacional sobre Drogas: publicaciones derivadas e impacto científico
}

\section{Financed research from Government Delegation grants for the National Plan on Drugs: research assessment and scientific impact}

\author{
Rafael Aleixandre-Benavent*,***, Víctor Agulló-Calatayud*,****, \\ Adolfo Alonso-Arroyo***, Francisco-Jesús Bueno-Cañigral*****, Lourdes \\ Castelló-Cogollos******, Rut Lucas-Domínguez***, David Melero-Fuentes******, \\ Andrea Sixto-Costoya***, Antonio Vidal-Infer***, Juan-Carlos Valderrama-Zurián****. \\ * Unidad de Investigación e Información Social y Sanitaria-UISYS. CSIC-Universitat de València. \\ ** Departamento de Historia de la Ciencia y Documentación. Universitat de València. \\ *** Ingenio. CSIC-Universitat Politècnica de València. \\ **** Departamento de Sociología y Antropología Social. Universitat de València. \\ ***** Servicio de Drogodependencias (PMD/UPCCA-València). Concejalía de Salud y Consumo. Ajuntament de València. \\ ****** Facultad de Psicología. Instituto de Documentación y Tecnologías de la Información.
}

Universidad Católica de Valencia San Vicente Mártir.

\section{Resumen}

Los trastornos adictivos son un grave problema de salud al que se des-
tinan gran cantidad de recursos de investigación. El propósito de este
trabajo es analizar la evolución e impacto científico de las publicacio-
nes derivadas de las ayudas a proyectos de investigación financiados
por el Plan Nacional Sobre Drogas (PNSD). La relación de ayudas
concedidas fue proporcionada por el PNSD. Las publicaciones deri-
vadas se obtuvieron preguntando a los investigadores principales de
las ayudas y buscando en Web of Science y Scopus. Se calcularon indi-
cadores bibliométricos y tendencias evolutivas de la producción cien-
tífica por proyecto. Por término medio, el PNSD concedió 15 ayudas
anuales a proyectos de investigación, con un importe anual cercano
al millón de euros (944.200,64€) y un importe medio por ayuda de
algo más de $60.000 €$, siendo mayor en la investigación básica y en al-
cohol. El 71,9\% de las ayudas tuvieron publicaciones derivadas y casi
la mitad produjeron entre una y tres publicaciones, siendo la investi-
gación básica la más prolífica. La revista extranjera en la que más ar-
tículos se publicaron fue Psychopharmacology (50) y entre las españolas
destacó Adicciones (28). Se identificó un alto índice de coautoría y de
colaboración internacional. La mayoría de los proyectos financiados

\begin{abstract}
Addictive disorders are a serious health problem to which large amounts of research resources are devoted. This study aims to analyze the evolution and scientific impact of the publications derived from the funding of research projects by the Spanish National Plan on Drugs (PNSD). The list of grants awarded was provided by the PNSD. Derived publications were obtained by asking the principal investigators of the grants and searching in the Web of Science and Scopus. Bibliometric indicators and evolutive trends of scientific production per project were calculated. On average, the PNSD conferred 15 annual grants to research projects, with an annual amount close to one million euros (€944,200.64) and an average amount per grant of just over $€ 60,000$, being higher in basic research and in alcohol. $71,9 \%$ of the grants had derived publications and almost half of them produced between one and three publications, with basic research being the most prolific. The international journal in which most articles were published was Psychopharmacology (50) and among Spanish journals, Adicciones stood out (28). A high level of co-authorship and international collaboration was identified. Most of the PNSD-funded projects produced research articles, many of them in journals belonging to the
\end{abstract}


por el PNSD produjeron artículos de investigación y muchos de ellos en revistas del primer y segundo cuartil del Journal Citation Reports. Los resultados de este estudio han permitido conocer la repercusión científica de las ayudas a proyectos de investigación del PNSD y puede contribuir a determinar futuras prioridades de financiación.

Palabras clave: Trastornos por abuso de sustancias; Ayudas económicas; Proyectos de investigación; Bibliometría; Publicaciones derivadas. ace casi cinco décadas que el consumo de drogas y las consecuencias que conlleva entró a formar parte de las principales preocupaciones del ciudadano español. El problema suscitó diferentes debates políticos, sanitarios y sociales, requiriendo, una respuesta gubernamental que se produjo en el Consejo de Ministros del 24 de julio de 1985, fecha en la que se creó el Plan Nacional Sobre Drogas con el fin de potenciar y coordinar las políticas de drogodependencias de las administraciones públicas. En su declaración de principios, el apartado 4 se refería a la investigación en drogodependencias en los siguientes términos: «la investigación permanente que debe ser potenciada tanto en lo que se refiere al conocimiento de la realidad de manera que facilite la planificación y desarrollo de acciones acordes con la misma, como en lo que corresponde a investigaciones aplicadas, que permitan delimitar nuevas experiencias de prevención y tratamiento. Asimismo, en el campo de la investigación urge —en nuestro país- desarrollar métodos normalizados que hagan posible comparar y completar los resultados obtenidos, en diferentes trabajos y en diferentes épocas» (Real Decreto 1677/1985).

Han pasado 35 años desde esa declaración de principios y, con la perspectiva que da el tiempo transcurrido, se puede decir que hoy la investigación en drogodependencias es una realidad firmemente asentada en nuestro país desde hace tiempo, tal y como se observa en varios estudios bibliométricos que analizaron la investigación española en el área (Aleixandre, 1999; Álvarez y del Río, 2003; Ballesteros, Torrens y Valderrama, 2006; González-Alcaide, Calafat, Becoña, Thijs y Glänzel, 2016; Rodríguez de Fonseca et al., 2006). Uno de los principales logros es la consolidación de una convocatoria anual de subvenciones a proyectos de investigación del Plan Nacional sobre Drogas que, fundamentada en el criterio de calidad de los proyectos presentados en concurrencia competitiva, ha propiciado la subvención estatal de un número muy apreciable de proyectos, así como un mayor seguimiento y control de la administración sobre el cumplimiento de los objetivos por los investigadores principales (Plan Nacional sobre Drogas, s.f.a). También se han instaurado otros tipos de subvenciones como, por ejemplo, ayudas económicas a entidades privadas sin fines de lucro para la realización de programas sobre adicciones con cargo al Fondo de Bienes first and second quartiles of the Journal Citation Reports. The results of this study have revealed the scientific impact of the PNSD research projects funding and may contribute to determining future funding priorities.

Keywords: Substance-related disorders; Funding support; Research projects; Bibliometrics; Publications resulting.

decomisados por tráfico ilícito de drogas y otros delitos relacionados, en aplicación de la Ley 17/2003; ayudas para la realización de programas supracomunitarios sobre adicciones (Ministerio de Sanidad, Servicios Sociales e Igualdad, 2019); ayudas a corporaciones locales para el desarrollo de programas de prevención de las drogodependencias, con cargo al Fondo de bienes decomisados por tráfico ilícito de drogas y otros delitos relacionados (Plan Nacional sobre Drogas, s.f.b).

Una faceta importante en la evaluación de la investigación es cuantificar el rendimiento de la investigación financiada con fondos públicos o privados. El objetivo de este trabajo es conocer los proyectos financiados por el PNSD desde el año 2000 hasta el 2016 y evaluar el rendimiento académico de las ayudas mediante el análisis bibliométrico de las publicaciones derivadas de estos proyectos en revistas científicas.

\section{Métodos}

\section{Obtención de los datos de los proyectos}

La lista de las ayudas a proyectos de investigación financiados desde el año 2000 a 2016 por el PNSD se obtuvo de la página web http://www.pnsd.mscbs.gob.es/delegacionGobiernoPNSD/convocatoriaSubvenciones/ongs/ proyecInvestig.htm. En 77 ayudas a proyectos anteriores a 2004, se disponía información sobre la institución del investigador principal pero no sobre quién era este investigador, ya que su nombre no estaba registrado. Se recogió la siguiente información de los proyectos: nombre del investigador principal, institución, cuantía de la ayuda y año de concesión. Los proyectos se clasificaron según: a) sustancias de las que trataba, clasificándose en: alcohol, alucinógenos, cannabis, cocaína, drogas de síntesis, opiáceos, psicoestimulantes, tabaco y drogas en general; b) tipo de investigación: básica, clínica-psiquiatría, documentación, genética, medicina general, políticas públicas, prevención, radiología, salud pública, servicios sociales, tratamiento, urgencias.

\section{Búsqueda de las publicaciones derivadas de las ayudas}

Para conocer las publicaciones derivadas se realizó un buzoneo electrónico a los investigadores principales de cada ayuda en el que se les solicitaban las referencias bi- 
bliográficas de las publicaciones derivadas de los proyectos financiados. Cuando los investigadores principales no proporcionaron estas referencias, se buscaron en la Colección Principal Web of Science (WoS) y Scopus, combinando las palabras clave del título del proyecto con los apellidos de los investigadores principales. Estas fuentes son las dos bases de datos de referencia comúnmente empleadas en la evaluación de la actividad científica y en la determinación de indicadores bibliométricos, sobre todos los basados en las citas que reciben los trabajos publicados (Bar-Ilan, Levene y Lin, 2007; Marx, Schier y Wanitschek, 2001).

En las 77 ayudas en las que no constaba el investigador principal, se identificó la producción derivada a partir del título del proyecto. También se realizó una búsqueda bibliográfica en el campo «Entidad financiadora» de WoS y Scopus. La ecuación de búsqueda incluía los términos («spanish national drug*» $O R$ «pnd» $O R$ «pnsd» $O R$ «plan nacional de drogas» $\mathrm{OR}$ «plan nacional sobre drogas» $\mathrm{OR}$ «plan nacional en drogas» $\mathrm{OR}$ «plan nacional drogas» OR «national drug plan» OR «National Plan on Drugs» OR ((Nacional OR Plan OR National) AND (drug* or droga*)) y se filtró a los artículos y revisiones. Los resultados obtenidos fueron cotejados por cuatro de los autores, especialistas en el campo de los trastornos adictivos y la documentación científica, para determinar si cada trabajo recuperado se correspondía realmente con el proyecto al que estaba asignado. Para que un artículo fuera considerado como publicación derivada tenía que cumplir dos criterios: a) el investigador principal del proyecto debía formar parte de los autores del artículo; b) la fecha de publicación del artículo debía ser posterior o en el mismo año a la de la concesión de la ayuda. Las búsquedas de los artículos derivados se realizaron entre septiembre y diciembre de 2019 y se incluyeron todas las publicaciones derivadas de los proyectos de investigación incluidas en las bases de datos a 31 de diciembre de 2019. Por lo tanto, se ha realizado una espera de al menos 3 años desde la última convocatoria del PNSD incluida en este trabajo, tiempo que, de acuerdo con Kingwell et al (2006), se considera suficiente para que se publiquen los resultados de las investigaciones y minimizar posibles pérdidas. El número de citas de cada publicación derivada fue consultado el 6 de abril de 2020 para que estuvieran lo más actualizadas posible.

\section{Extracción de datos e indicadores}

De cada artículo derivado se extrajo la siguiente información: autores, título del artículo, revista, número de citas recibidas y año de publicación. Estos datos se completaron con la siguiente información complementaria de las revistas a partir de la edición 2018 del Journal Citation Reports (jcr.clarivate.com): país de publicación y factor de impacto de cinco años.

Posteriormente se obtuvieron los siguientes indicadores: evolución anual del número de ayudas, importe anual e importe medio por ayuda; sustancia que se estudia en el proyecto de investigación y tipo de investigación financiada; evolución anual de las ayudas según hayan o no publicaciones derivadas; años transcurridos desde la concesión de las ayudas y las publicaciones derivadas; evolución anual del número de artículos publicados en revistas españolas y extranjeras; instituciones beneficiarias de las ayudas y número de artículos publicados; revistas en las que se publicaron los artículos, número de citas recibidas, índices de colaboración de autores, existencia de colaboración nacional e internacional y mapa de colaboración entre los países de las instituciones de los autores. El análisis de citas se utiliza para evaluar la influencia académica de los trabajos publicados en un campo temático (Moed, 2009; Waltman, 2016) y se ha convertido en una herramienta útil y de uso habitual, ya que la frecuencia con la que se citan los trabajos pueden considerarse un parámetro objetivo de su calidad (Bornmann y Leydesdorff, 2014; Byrne y Chapman, 2005; Mahabee-Gittens, Gordon, Melink y Merianos, 2017; Marx et al., 2001; Tanner-Smith y Polanin, 2016; van Wesel, 2015).

Para el análisis estadísticos se utilizó la versión 26 de SPSS (IBM, Armonk, NY, USA). Se realizaron análisis descriptivos y de frecuencias, chi-cuadrado $\left(\chi^{2}\right)$ para la comparación de variables categóricas y prueba no paramétrica de Kruskal-Wallis (K-W) para variables cuantitativas de diversas categorías. El nivel de significación estadística se estableció en $\mathrm{p}<0.05$. En el caso del tipo de investigación se agruparon las diversas categorías en tres grandes áreas de investigación: básica, ciencias de la salud (clínica-psiquiatría, genética, medicina general, radiología, salud pública, tratamiento, urgencias) y ciencias sociales (documentación, políticas públicas, prevención, servicios sociales).

Para la llevar a cabo la representación de la red de colaboración internacional en las publicaciones derivadas se utilizó el software Pajek, que integra el algoritmo de poda Pathfinder para reducir el espacio dimensional y el algoritmo Kamada-Kawai como algoritmo de visualización. Esta red se ha mostrado en forma de mapa geográfico mundial. En ella, el diámetro de las esferas es proporcional al número de países distintos con los que se relaciona cada país y el ancho de las líneas representa el número de veces que dos países colaboran. La red se ha formado aplicando con un umbral de más de un artículo publicado en colaboración.

Los datos de los que se extrajeron los resultados así como las tablas y figuras adicionales están disponibles como material suplementario en Zenodo (Aleixandre-Benavent et al., 2020a; Aleixandre-Benavent et al., 2020b; Aleixandre-Benavent et al., 2020c).

\section{Resultados}

El PNSD ha convocado ayudas para el desarrollo de proyectos de investigación sobre adicciones desde el año 
2000. En la tabla 1 puede apreciarse la distribución anual de las 253 ayudas concedidas desde el año 2000 hasta 2016 por un importe total de 16.051.410,87€. Para disponer de valores homogéneos en todo el periodo, los importes de las ayudas correspondientes a los años 2000 y 2001, que se publicaron en pesetas (107.700.000 pesetas en $2000 \mathrm{y}$ 75.250.000 pesetas en 2001), se han convertido en euros (asignándoles el valor del cambio oficial en 2002, que fue de 166,386 pesetas por euro). El año en el que más ayudas se concedieron fue 2005 (21 ayudas), mientras que en 2012 solo se concedieron diez. Por término medio se concedieron alrededor de 15 ayudas anuales, con un importe medio anual cercano al millón de euros $(944.200,64 €)$ y un importe medio por ayuda de un poco más de 60.000€. Como puede apreciarse, ni el número de ayudas ni su importe muestran a lo largo del tiempo una tendencia creciente, pues existen variaciones anuales. Aunque en los tres últimos años ha aumentado el número de ayudas (de 11 ayudas en 2014 a 17 en 2016), el importe concedido se ha reducido en un 27\% (de 1.117.951€ en 2014 a $815.286 €$ en 2016). Paralelamente, el importe medio por ayuda descendió casi a la mitad de 2014 a 2016.

El $36 \%$ de los proyectos estaban centrados en el alcohol, el $22,9 \%$ en drogas en general y el $19 \%$ en la cocaína (tabla 1 de material suplementario (MS)) sólo un proyecto trataba específicamente sobre alucinógenos $(0,4 \%)$. Excepto en un año siempre se han financiado proyectos de alcohol o drogas en general. Desde 2010 se han financiado fundamentalmente proyectos sobre alcohol representando anualmente entre un 52,9\%, año 2016, y un 83,3\%, año 2015, de los proyectos. Los dos proyectos con más financiación trataban sobre psicoestimulantes (194.087€) y cannabis $(193.357 €)$, siendo los proyectos de drogas de síntesis los que más financiación obtenían (mediana: 68.943€). Se han obtenido diferencias estadísticamente significativas en la cuantía de los importes según el tipo de sustancias de investigación (K-W: $\left.\chi^{2}=20,04 ; \mathrm{p}<0,006\right)$ (figura 1a).

La investigación básica representa el 42,7\% de los proyectos, seguido de los estudios en el área de las ciencias salud, y entre ellos los centrados en la clínica del área de la psiquiatría y la psicología. Los proyectos de prevención representan el 9,5\% del total de proyectos. Entre las áreas menos financiadas destacan las de aspectos sociales, medicina general, estudios radiológicos cerebrales, urgencias y políticas públicas (tabla 2, MS). Se observan diferencias en la cuantía de los importes entre las áreas de investigación (K-W: $\left.\chi^{2}=20,7 ; \mathrm{p}<0,001\right)$, siendo los proyectos del área de básica los que reciben más financiación (figura 1b).

La figura 2 permite observar la evolución anual de los proyectos con artículos derivados en números absolutos. Se aprecia un comportamiento casi paralelo de las ayudas con publicación derivada y sin ella al principio de la serie (desde el año 2000 a 2004), y el despegue del número de

Tabla 1. Evolución anual de las cuantías en euros de las Ayudas de la Delegación del Gobierno para el Plan Nacional sobre Drogas para el desarrollo de proyectos de investigación sobre adicciones.

\begin{tabular}{|c|c|c|c|c|c|c|c|}
\hline Año & № ayudas & $\begin{array}{l}\text { Total de importe } \\
\text { de inversión } \\
(€)\end{array}$ & $\begin{array}{l}\text { Media importe } \\
\text { por ayudas } \\
(€)\end{array}$ & $\begin{array}{l}\text { Desviación } \\
\text { estándar } \\
(€)\end{array}$ & $\begin{array}{c}\text { Media recortada } \\
\text { al } 5 \% \\
(€)\end{array}$ & $\underset{(€)}{\text { Mínimo }}$ & $\begin{array}{l}\text { Máximo } \\
\text { (€) }\end{array}$ \\
\hline 2000 & 16 & $647.290,04$ & $40.455,63$ & $21.524,54$ & $39.508,20$ & $10.818,21$ & $87.146,76$ \\
\hline 2001 & 16 & $452.261,61$ & $28.266,35$ & $19.095,57$ & $27.433,70$ & $3.005,06$ & $68.515,38$ \\
\hline 2002 & 17 & $866.124,92$ & $50.948,52$ & $40.555,07$ & $48.998,36$ & 4.000 & 133.000 \\
\hline 2003 & 20 & $1.380 .925,31$ & $69.046,27$ & $44.164,66$ & $65.316,02$ & 11.150 & 194.087 \\
\hline 2004 & 14 & 655.510 & $46.822,14$ & $47.827,73$ & $40.526,38$ & 13.611 & 193.357 \\
\hline 2005 & 21 & 1.158 .149 & $55.149,95$ & $36.242,69$ & $54.224,42$ & 11.400 & 115.630 \\
\hline 2006 & 13 & 893.366 & $68.720,46$ & $23.318,05$ & $68.466,90$ & 35.000 & 107.005 \\
\hline 2007 & 14 & 1.089 .010 & $77.786,43$ & $44.316,25$ & $77.809,43$ & 8.000 & 147.160 \\
\hline 2008 & 13 & 772.610 & $59.431,54$ & $31.602,93$ & $58.615,60$ & 10.550 & 123.000 \\
\hline 2009 & 15 & 975.700 & $65.046,67$ & $33.703,78$ & $64.829,63$ & 16.000 & 118.000 \\
\hline 2010 & 16 & 1.214 .490 & $75.905,63$ & $33.028,72$ & $74.228,47$ & 20.000 & 162.000 \\
\hline 2011 & 15 & 1.134 .821 & $75.654,73$ & $22.673,36$ & $74.621,09$ & 43.915 & 126.000 \\
\hline 2012 & 10 & 918.200 & 91.820 & $38.422,15$ & $90.661,11$ & 35.300 & 169.200 \\
\hline 2013 & 13 & 939.060 & $72.235,38$ & $38.981,04$ & $71.598,26$ & 15.793 & 140.146 \\
\hline 2014 & 11 & 1.117 .951 & $101.631,91$ & $37.484,70$ & $100.064,68$ & 57.836 & 173.638 \\
\hline 2015 & 12 & 1.020 .656 & $85.054,67$ & $26.264,88$ & $83.401,24$ & 54.844 & 145.027 \\
\hline 2016 & 17 & 815.286 & 47.958 & $19.905,50$ & $47.914,56$ & 12.698 & 84.000 \\
\hline Total & 253 & $16.051 .410,87$ & $63.444,31$ & $37.514,36$ & $61.172,43$ & $3.005,06$ & 194.087 \\
\hline
\end{tabular}




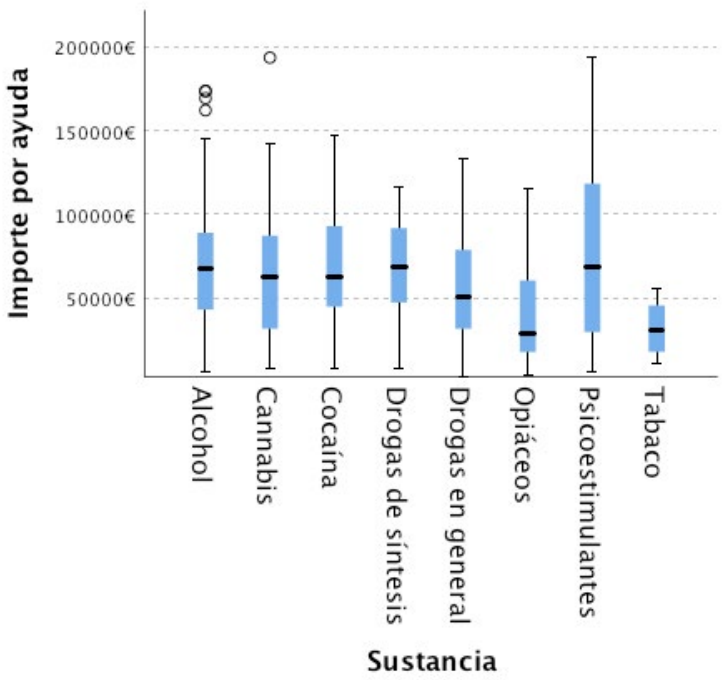

Figura 1a. Importe de la cuantía por sustancia de investigación. Prueba de Kruskal-Wallis (p<o.006). Al estar financiado solamente un proyecto sobre alucinógenos, este se ha excluido del análisis.

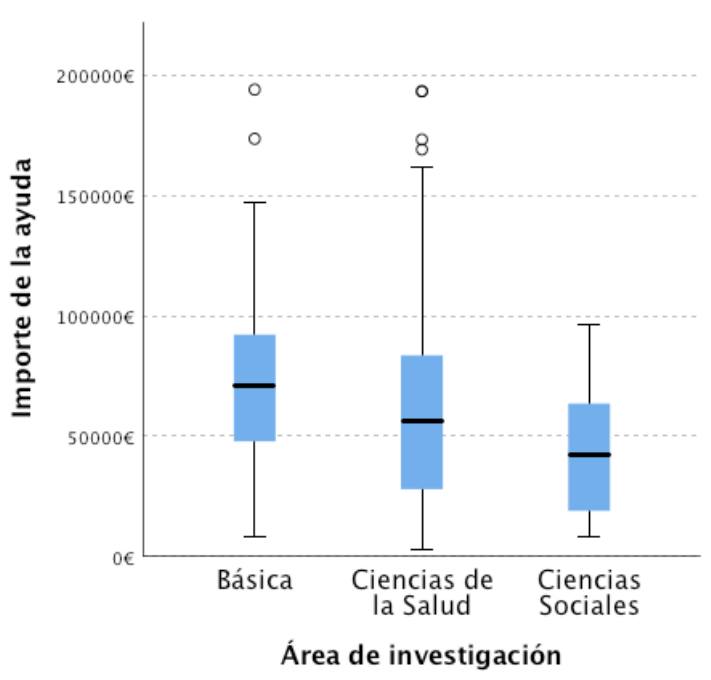

Figura $1 b$. Distribución del importe de las ayudas por área de investigación. Prueba de Kruskal-Wallis (p<0.001).

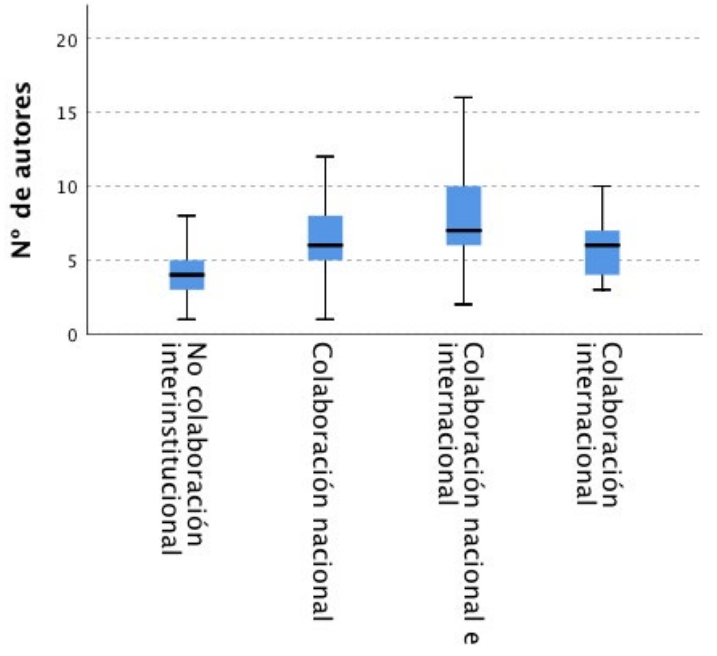

Tipo de Colaboración

Figura 1c. Distribución del número de autores por tipo de colaboración. Prueba de Kruskal-Wallis (p<o.001).

Tabla 2. Publicaciones derivadas según área de investigación y tipo de sustancia.

\begin{tabular}{lccccc}
\hline & \multicolumn{5}{c}{ Número de publicaciones derivadas } \\
\hline Área de investigación & Mínimo & Máximo & Mediana & $\begin{array}{c}\text { Media } \\
\text { recortada al 5\% }\end{array}$ & Prueba de Kruskal-Wallis \\
\hline Básica & 0 & 19 & 4 & 4,58 & \\
Ciencias de la salud & 0 & 27 & 2 & 3,02 & $\chi^{2}=14,99$ p $<0,002$ \\
Ciencias Sociales & 0 & 15 & 1 & 1,93 & \\
\hline Sustancia & 0 & & & & \\
\hline Alcohol & 0 & 27 & 2,5 & 4,11 & \\
Cannabis & 0 & 24 & 3 & 4,38 & \\
Cocaína & 0 & 11 & 2 & 3,57 & \\
Drogas de síntesis & 0 & 18 & 1 & 1,94 & \\
Drogas en general & 0 & 17 & 1 & 2,20 & \\
Opiáceos & 0 & 13 & 4 & 5,94 & \\
Psicoestimulantes & 0 & 7 & 1 & 1,97 & \\
Tabaco & & & & & \\
\hline
\end{tabular}




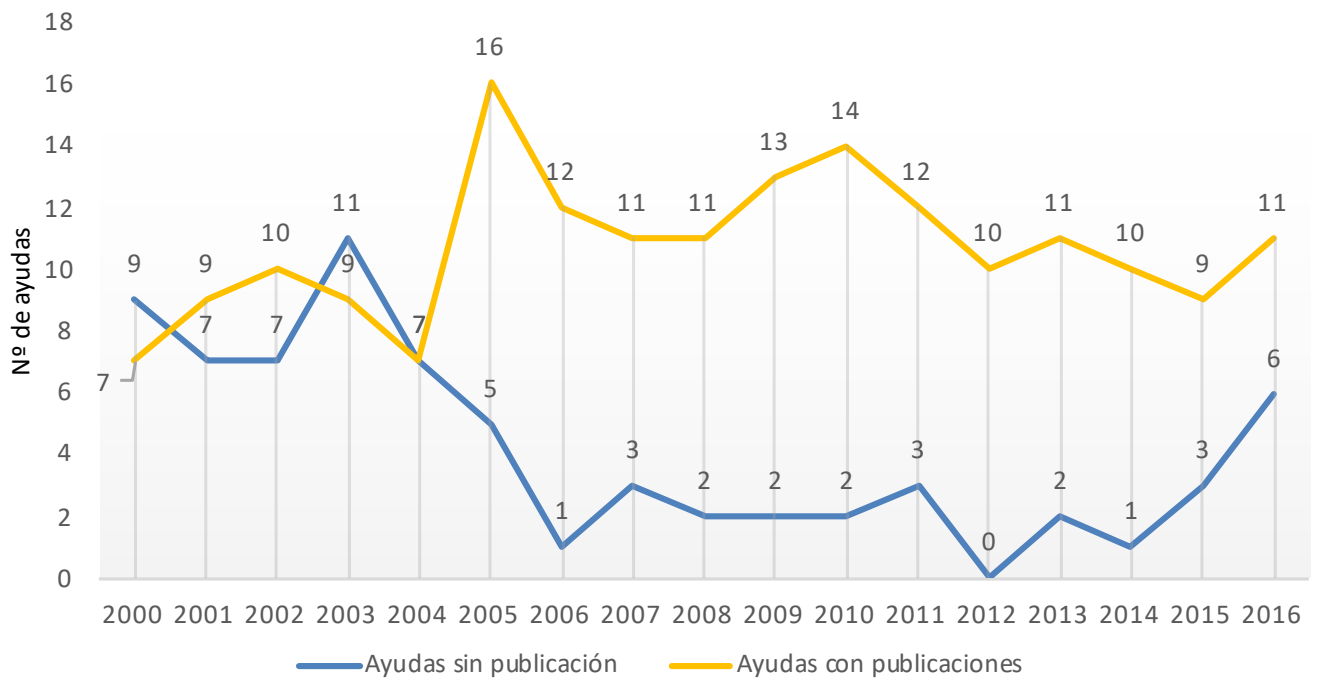

Figura 2. Evolución anual del número de Ayudas de la Delegación del Gobierno para el Plan Nacional sobre Drogas con publicación derivada y sin ella.

ayudas con publicaciones derivadas a partir de 2005. El año en el que todos los proyectos tuvieron al menos una publicación subsiguiente fue $2012(100 \%)$ mientras el año 2000 obtuvo el menor porcentaje $(43,8 \%)$.

El número de publicaciones derivadas por las 182 ayudas que han dado lugar a publicaciones científicas se muestra en la figura $1 \mathrm{MS}$. Del total de ayudas ( $\mathrm{n}=253), 84$ $(33,2 \%)$ han producido una, dos o tres publicaciones derivadas, predominan las ayudas que han dado lugar a una única publicación derivada (32 ayudas; $12,6 \%$ ), seguido de las ayudas con dos publicaciones (31 ayudas, $12,3 \%$ ) y de las ayudas con tres publicaciones (22 ayudas; $8,7 \%$ ). Las ayudas con más de 14 publicaciones representan el 4,4\%. El número total de publicaciones derivadas fue de $1.019 \mathrm{si}$ bien en 31 constaban dos ayudas del PNSD como fuente de financiación. Los proyectos del área de investigación básica presentan una mediana de publicaciones derivadas mayor que los de ciencias de la salud y que los de ciencias sociales $\left(\mathrm{K}-\mathrm{W}: \chi^{2}=14,99 ; \mathrm{p}<0.002\right)$ (tabla 2 ). Si se consideran las sustancias que se investiga en el proyecto de investigación, son los proyectos sobre psicoestimulantes los que presentan más publicaciones derivadas. Se observa diferencias estadísticamente significativas en el número de publicaciones derivadas de los proyectos sobre cocaína o alcohol respecto a los de drogas en general (tabla 2). La mediana de publicaciones derivadas de los proyectos sobre opiáceos es de un artículo; esta mediana tiene el mismo valor en los proyectos sobre tabaco y drogas en general.

También es relevante conocer el tiempo transcurrido desde la concesión de las ayudas y las publicación derivadas (figura 2, MS). La mayor parte de los artículos se publicaron a los tres años $(21,5 \%)$ y a los cuatro años $(19 \%)$. El porcentaje de publicaciones el mismo año que el de concesión del proyecto fue del 3,3\%. La publicación a partir de los seis años es del $(11,2 \%)$.
El número de artículos anuales publicados en revistas españolas y extranjeras se presenta en la tabla 3 de MS. De los 1.019 artículos, 937 (92\%) se publicaron en revistas extranjeras y $82(8 \%)$ en revistas españolas.

La tabla 3 ofrece las revistas en las que se han publicado cinco o más trabajos derivados de las ayudas. Las revistas destinatarias de un mayor número de trabajos fueron Psychopharmacology $(\mathrm{n}=50)$, seguida de Drug and Alcohol Dependence $(\mathrm{n}=38)$ y Addiction Biology $(\mathrm{n}=35)$. La primera revista española fue Adicciones $(\mathrm{n}=28)$. En número de citas en WoS el ranking está liderado por Drug and Alcohol Dependence $(\mathrm{n}=1.243)$. La revista con un mayor indicador «citas por trabajo» en WoS fue Biological Psychiatry $(\mathrm{C} / \mathrm{T}=$ $74,25)$. De las 52 revistas en las que se ha publicado cinco o más trabajos, 26 (50\%) están en el primer cuartil de JCR y $17(32,7 \%)$ en el segundo cuartil. Se han publicado 603 trabajos en revistas de primer o segundo cuartil.

Las 70 instituciones beneficiarias de las Ayudas de la Delegación del Gobierno para el Plan Nacional sobre Drogas se presentan en la tabla 4 . De ellas, 14 no reportaron publicaciones derivadas. Las que más ayudas obtuvieron durante el periodo analizado fueron la Universidad Complutense de Madrid ( $\mathrm{n}=21$ ), seguida de la Fundación Instituto Mar de Investigaciones Médicas (IMIM) $(\mathrm{n}=18)$, el Consejo Superior de Investigaciones Científicas $(n=15)$ y las universidades de Santiago de Compostela $(n=13)$ y València $(n=12)$. Las cinco instituciones que recibieron un mayor importe total fueron las mismas que se han mencionado antes. En número absoluto de trabajos derivados de las ayudas destacan la Universidad Complutense de Madrid $(\mathrm{n}=79)$, seguida de la Universitat Pompeu Fabra $(\mathrm{n}=63)$ y la Universitat de València $(\mathrm{n}=60)$. El indicador «número de trabajos por ayuda» es mayor para la Fundación Pública Marqués de Valdecilla $(n=27)$, seguida de Instituto de Investigación Sanitaria Fundación Jiménez Díaz $(n=18)$ 
Rafael Aleixandre-Benavent, Víctor Agulló-Calatayud, Adolfo Alonso-Arroyo, Francisco-Jesús Bueno-Cañigral, Lourdes Castelló-Cogollos, Rut Lucas-Domínguez, David Melero-Fuentes, Andrea Sixto-Costoya, Antonio Vidal-Infer, Juan-Carlos Valderrama-Zurián

Tabla 3. Revistas con más de 5 trabajos derivados y número de citas (Ordenado por número de trabajos derivados y número de citas totales).

\begin{tabular}{|c|c|c|c|c|c|c|}
\hline Revista & País & $\begin{array}{c}\text { № trabajos } \\
\text { derivados }\end{array}$ & $\begin{array}{c}\text { № de Citas } \\
\text { totales }\end{array}$ & $\begin{array}{l}\text { Citas / Trabajos } \\
\text { en Wos }\end{array}$ & FI 5 años & Cuartiles \\
\hline Psychopharmacology & Alemania & 50 & 1.075 & 21,5 & 3,3 & Q2 \\
\hline Drug and Alcohol Dependence & Suiza & 38 & 1.243 & 32,71 & 3,989 & Q1 \\
\hline Addiction Biology & Inglaterra & 35 & 581 & 16,6 & 4,02 & Q1 \\
\hline Neuropharmacology & Inglaterra & 34 & 807 & 23,74 & 4,494 & Q1 \\
\hline Plos One & Estados Unidos & 32 & 673 & 21,03 & 3,337 & Q2 \\
\hline Adicciones* & España & 28 & 225 & 9,78 & 2,221 & Q1 \\
\hline British Journal of Pharmacology & Inglaterra & 23 & 643 & 27,96 & 5,755 & Q1 \\
\hline Journal of Psychopharmacology & Inglaterra & 23 & 332 & 14,43 & 4,434 & Q1 \\
\hline Neuropsychopharmacology & Inglaterra & 22 & 1.104 & 50,18 & 6,705 & Q1 \\
\hline European Neuropsychopharmacology & Países Bajos & 19 & 332 & 17,47 & 4,394 & Q1 \\
\hline Journal of Neurochemistry & Inglaterra & 16 & 829 & 51,81 & 4,371 & Q1 \\
\hline International Journal of Neuropsychopharmacology & Inglaterra & 15 & 223 & 14,87 & 4,39 & Q1 \\
\hline Neuroscience & Inglaterra & 14 & 430 & 30,71 & 3,504 & Q2 \\
\hline Alcoholism-clinical and Experimental Research & Estados Unidos & 14 & 328 & 23,43 & 3,276 & Q2 \\
\hline Progress in Neuro-psychopharmacology \& Biological Psychiatry & Inglaterra & 14 & 235 & 16,79 & 4,159 & Q1 \\
\hline European Journal of Pharmacology & Países Bajos & 12 & 194 & 16,17 & 3,12 & Q2 \\
\hline Gaceta Sanitaria & España & 12 & 140 & 11,67 & 1,918 & Q2 \\
\hline Psychiatry Research & Países Bajos & 12 & 128 & 10,67 & 2,67 & Q2 \\
\hline Scientific Reports & Inglaterra & 11 & 54 & 4,91 & 4,525 & Q1 \\
\hline Alcohol and Alcoholism & Inglaterra & 10 & 556 & 55,6 & 2,882 & Q2 \\
\hline Pharmacology Biochemistry and Behavior* & Inglaterra & 10 & 266 & 29,55 & 2,867 & Q2 \\
\hline Schizophrenia Research* & Países Bajos & 10 & 233 & 25,88 & 4,583 & Q1 \\
\hline Addictive Behaviors & Inglaterra & 10 & 220 & 22 & 3,325 & Q1 \\
\hline Behavioural Pharmacology & Estados Unidos & 10 & 196 & 19,6 & 1,869 & Q3 \\
\hline Neuroscience Letters & Países Bajos & 10 & 155 & 15,5 & 2,228 & Q3 \\
\hline Behavioural Brain Research & Países Bajos & 10 & 154 & 15,4 & 3,021 & Q2 \\
\hline Neurobiology of Disease & Inglaterra & 9 & 434 & 48,22 & 5,304 & Q1 \\
\hline Neuroscience and Biobehavioral Reviews & Inglaterra & 9 & 393 & 43,67 & 9,42 & Q1 \\
\hline Addiction & Inglaterra & 9 & 202 & 22,44 & 6,45 & Q1 \\
\hline Frontiers in Behavioral Neuroscience & Suiza & 9 & 145 & 16,11 & 3,454 & Q2 \\
\hline Biological Psychiatry & Estados Unidos & 8 & 594 & 74,25 & 11,275 & Q1 \\
\hline European Addiction Research & Suiza & 8 & 67 & 8,38 & 2,532 & Q2 \\
\hline Frontiers in Psychiatry & Suiza & 8 & 41 & 5,13 & $\mathrm{n} / \mathrm{a}$ & Q2 \\
\hline Journal of Psychiatric Research & Inglaterra & 7 & 225 & 32,14 & 4,475 & Q1 \\
\hline Physiology \& Behavior & Estados Unidos & 7 & 136 & 19,43 & 2,848 & Q2 \\
\hline Biochemical Pharmacology & Estados Unidos & 7 & 50 & 7,14 & 4,637 & Q1 \\
\hline Substance Use \& Misuse & Estados Unidos & 7 & 42 & 6 & 1,584 & Q4 \\
\hline Journal of Affective Disorders & Países Bajos & 7 & 39 & 5,57 & 4,16 & Q1 \\
\hline Psicothema & España & 6 & 104 & 17,33 & 2,057 & Q2 \\
\hline European Journal of Neuroscience & Inglaterra & 5 & 240 & 48 & 3,023 & Q3 \\
\hline Alcohol & Estados Unidos & 5 & 220 & 44 & 2,528 & Q3 \\
\hline Cerebral Cortex & Estados Unidos & 5 & 218 & 43,6 & 6,149 & Q1 \\
\hline Brain Behavior and Immunity & Estados Unidos & 5 & 215 & 43 & 6,616 & Q1 \\
\hline Psychological Medicine & Estados Unidos & 5 & 196 & 39,2 & 6,313 & Q1 \\
\hline Brain Research & Países Bajos & 5 & 151 & 30,2 & 2,937 & Q2 \\
\hline Current Pharmaceutical Design & $\begin{array}{c}\text { Emiratos Árabes } \\
\text { Unidos }\end{array}$ & 5 & 144 & 28,8 & 2,832 & Q3 \\
\hline Hormones and Behavior & Estados Unidos & 5 & 68 & 13,6 & 4,041 & Q1 \\
\hline International Journal of Environmental Research and Public Health & Suiza & 5 & 55 & 11 & 2,948 & Q1 \\
\hline Journal of Substance Abuse Treatment & Estados Unidos & 5 & 52 & 10,4 & 2,753 & Q2 \\
\hline Actas Españolas de Psiquiatría & España & 5 & 40 & 8 & 1,318 & Q3 \\
\hline International Journal of Drug Policy & Países Bajos & 5 & 40 & 8 & 4,45 & Q3 \\
\hline Trastornos Adictivos $* \star$ & España & 5 & - & - & $\mathrm{n} / \mathrm{a}$ & $\mathrm{n} / \mathrm{a}$ \\
\hline
\end{tabular}

Nota. *En estas revistas el número de citas por trabajo se calculó sobre el número de artículos que estaban incluidos en la Colección Principal de WoS. Adicciones $(n=23)$; Pharmacology Biochemistry and Behavior $(n=9)$; Schizophrenia Research $(n=9)$.

**Trastornos Aditivos no estaba incluida en la Colección Principal de Web of Science ni en el Journal Citation Reports. 
Repercusión científica de las ayudas de la Delegación del Gobierno para el Plan Nacional sobre Drogas: publicaciones derivadas e impacto científico

Tabla 4. Instituciones beneficiarias de las Ayudas de la Delegación del Gobierno para el Plan Nacional sobre Drogas y número de artículos derivados (Orden alfabético).

\begin{tabular}{|c|c|c|c|c|c|c|c|c|}
\hline Institución beneficiaria & $\begin{array}{c}\text { № de } \\
\text { ayudas }\end{array}$ & $\begin{array}{l}\text { № trabajos } \\
\text { derivados }\end{array}$ & Importe total & $\begin{array}{l}\text { Trabajos } \\
\text { derivados } \\
\text { por ayuda }\end{array}$ & $\begin{array}{l}\text { Importe por } \\
\text { trabajo }\end{array}$ & Citas & $\begin{array}{l}\text { Citas / } \\
\text { Trabajo }\end{array}$ & $\begin{array}{l}\text { Citas / } \\
\text { Ayuda }\end{array}$ \\
\hline Agència de Salud Pública de Barcelona & 5 & 16 & $304.787 €$ & 3 & $19.049,19 €$ & 390 & 24 & 78 \\
\hline Agencia Laín Entralgo & 1 & 0 & $57.000 €$ & 0 & No publicación & 0 & 0 & 0 \\
\hline $\begin{array}{l}\text { Asociación Colaboración Cochrane } \\
\text { Iberoamericana }\end{array}$ & 1 & 0 & $20.000 €$ & 0 & No publicación & 0 & 0 & 0 \\
\hline $\begin{array}{l}\text { Consejo Superior de Investigaciones } \\
\text { Científicas }\end{array}$ & 15 & 57 & $1.328 .112,53 €$ & 4 & $23.300,22 €$ & 2.591 & 45 & 173 \\
\hline Escuela Andaluza de Salud Pública & 1 & 0 & $80.000 €$ & 0 & No publicación & 0 & 0 & 0 \\
\hline Fundació Clínic per a la Recerca Biomèdica & 3 & 7 & $196.047 €$ & 2 & $28.006,71 €$ & 69 & 10 & 23 \\
\hline $\begin{array}{l}\text { Fundació Institut de Recerca de l'Hospital } \\
\text { Universitari Vall d'Hebron }\end{array}$ & 4 & 31 & $270.055 €$ & 8 & $8.711,45 €$ & 704 & 23 & 176 \\
\hline $\begin{array}{l}\text { Fundació Institut d'Investigació en Ciencias } \\
\text { de la Salut Germans Trias I Pujol }\end{array}$ & 4 & 23 & $287.643 €$ & 6 & $12.506,22 €$ & 54 & 2 & 14 \\
\hline $\begin{array}{l}\text { Fundació Privada Institut de Recerca } \\
\text { Biomèdica }\end{array}$ & 1 & 10 & $99.000 €$ & 10 & $9.900 €$ & 297 & 30 & 297 \\
\hline $\begin{array}{l}\text { Fundación Andaluza para la Atención a las } \\
\text { Drogodependencias }\end{array}$ & 2 & 5 & $124.770 €$ & 3 & $24.954 €$ & 140 & 28 & 70 \\
\hline Fundación Biomédica Galicia Sur & 1 & 0 & $33.925 €$ & 0 & No publicación & 0 & 0 & 0 \\
\hline $\begin{array}{l}\text { Fundación Canaria de Investigación Sanitaria } \\
\text { (FUNCANIS) }\end{array}$ & 1 & 0 & $12.698 €$ & 0 & No publicación & 0 & 0 & 0 \\
\hline $\begin{array}{l}\text { Fundación de la Comunidad Valenciana } \\
\text { Centro de Investigación Príncipe Felipe }\end{array}$ & 3 & 32 & $252.250 €$ & 11 & $7.882,81 €$ & 2.182 & 68 & 727 \\
\hline Fundación Hospital Alcorcón & 2 & 0 & $31.400 €$ & 0 & No publicación & & 0 & 0 \\
\hline Fundación Hospital Carlos Haya & 2 & 2 & $88.815,74 €$ & 1 & $44.407,87 €$ & 173 & 87 & 87 \\
\hline Fundación IDICHUS & 1 & 2 & $83.037 €$ & 2 & $41.518,50 €$ & 10 & 5 & 10 \\
\hline Fundación IMABIS & 4 & 36 & $335.000 €$ & 9 & $9.305,56 €$ & 820 & 23 & 205 \\
\hline $\begin{array}{l}\text { Fundación Instituto Mar de Investigaciones } \\
\text { Médicas (IMIM) }\end{array}$ & 18 & 51 & $1.650 .740,90 €$ & 3 & $32.367,47 €$ & 1.256 & 25 & 70 \\
\hline Fundación Miguel Servet & 1 & 14 & $23.000 €$ & 14 & $1.642,86 €$ & 262 & 19 & 262 \\
\hline $\begin{array}{l}\text { Fundación para la Formación e Investigación } \\
\text { Sanitaria de la Región de Murcia }\end{array}$ & 1 & 1 & $110.920 €$ & 1 & $110.920 €$ & 0 & 0 & 0 \\
\hline $\begin{array}{l}\text { Fundación para la Investigación Biomédica } \\
\text { del Hospital Universitario } 12 \text { de Octubre }\end{array}$ & 5 & 1 & $261.197,46 €$ & 0 & $261.197,46 €$ & 0 & 0 & 0 \\
\hline $\begin{array}{l}\text { Fundación para la Investigación Biosanitaria } \\
\text { de Andalucía Oriental Alejando Otero (FIBAO) }\end{array}$ & 1 & 2 & $8.000 €$ & 2 & $4.000 €$ & 50 & 25 & 50 \\
\hline $\begin{array}{l}\text { Fundación para la Investigación Sanitaria en } \\
\text { Castilla la Mancha }\end{array}$ & 2 & 1 & $28.525 €$ & 1 & $28.525 €$ & 129 & 129 & 65 \\
\hline Fundación Parc Científic de Barcelona & 1 & 0 & $70.100 €$ & 0 & No publicación & 0 & 0 & 0 \\
\hline Fundación Progreso y Salud & 1 & 2 & $53.650 €$ & 2 & $26.825 €$ & 17 & 9 & 17 \\
\hline $\begin{array}{l}\text { Fundación Pública Andaluza para la } \\
\text { Investigación de Málaga en Biomedicina y } \\
\text { Salud (FIMABIS) }\end{array}$ & 2 & 33 & $192.573 €$ & 17 & $5.835,55 €$ & 190 & 6 & 95 \\
\hline Fundación Pública Marqués de Valdecilla & 1 & 27 & $61.000 €$ & 27 & $2.259,26 €$ & 680 & 25 & 680 \\
\hline Fundación Universitaria San Pablo CEU & 6 & 11 & $359.481 €$ & 2 & $32.680,09 €$ & 54 & 5 & 9 \\
\hline $\begin{array}{l}\text { Fundación Valenciana de Investigaciones } \\
\text { Biomédica }\end{array}$ & 2 & 1 & $90.752,83 €$ & 1 & $90.752,83 €$ & 0 & 0 & 0 \\
\hline $\begin{array}{l}\text { Fundación Vasca de Innovación e } \\
\text { Investigación Sanitaria }\end{array}$ & 2 & 13 & $210.695 €$ & 7 & $16.207,31 €$ & 191 & 15 & 96 \\
\hline Hospital General Gregorio Marañón & 2 & 6 & $176.000 €$ & 3 & $29.333,33 €$ & 158 & 26 & 79 \\
\hline Hospital Universitario de Canarias & 1 & 0 & $13.611 €$ & 0 & No publicación & & 0 & 0 \\
\hline Hospital Universitario de Salamanca & 1 & 3 & $48.450 €$ & 3 & $16.150 €$ & 38 & 13 & 38 \\
\hline Hospital Universitario Ramón y Cajal & 1 & 3 & $14.350 €$ & 3 & $4.783,33 €$ & 51 & 17 & 51 \\
\hline $\begin{array}{l}\text { Institut de Recerca de l'Hospital de la Santa } \\
\text { Creu i Sant Pau }\end{array}$ & 10 & 10 & $301.849,35 €$ & 1 & $30.184,94 €$ & 117 & 12 & 12 \\
\hline
\end{tabular}


Rafael Aleixandre-Benavent, Víctor Agulló-Calatayud, Adolfo Alonso-Arroyo, Francisco-Jesús Bueno-Cañigral, Lourdes Castelló-Cogollos, Rut Lucas-Domínguez, David Melero-Fuentes, Andrea Sixto-Costoya, Antonio Vidal-Infer, Juan-Carlos Valderrama-Zurián

Tabla 4. Instituciones beneficiarias de las Ayudas de la Delegación del Gobierno para el Plan Nacional sobre Drogas y número de artículos derivados (Orden alfabético) (cont.).

\begin{tabular}{|c|c|c|c|c|c|c|c|c|}
\hline Institución beneficiaria & $\begin{array}{l}\text { № de } \\
\text { ayudas }\end{array}$ & $\begin{array}{c}\text { № trabajos } \\
\text { derivados }\end{array}$ & Importe total & $\begin{array}{c}\text { Trabajos } \\
\text { derivados } \\
\text { por ayuda }\end{array}$ & $\begin{array}{l}\text { Importe por } \\
\text { trabajo }\end{array}$ & Citas & $\begin{array}{l}\text { Citas / } \\
\text { Trabajo }\end{array}$ & $\begin{array}{l}\text { Citas / } \\
\text { Ayuda }\end{array}$ \\
\hline $\begin{array}{l}\text { Institut d'Investigacions Biomèdiques August } \\
\text { Pi i Sunyer }\end{array}$ & 1 & 6 & $82.980 €$ & 6 & $13.830 €$ & 63 & 11 & 63 \\
\hline $\begin{array}{l}\text { Instituto de Investigación Sanitaria Fundación } \\
\text { Jiménez Díaz }\end{array}$ & 1 & 18 & $70.773 €$ & 18 & $3.931,83 €$ & 54 & 3 & 54 \\
\hline Instituto de Salud Carlos III & 2 & 8 & $114.314 €$ & 4 & $14.289,25 €$ & 237 & 30 & 119 \\
\hline Universidad Autónoma de Madrid & 2 & 22 & $335.284 €$ & 11 & $15.240,18 €$ & 195 & 9 & 98 \\
\hline Universidad Castilla la Mancha & 1 & 4 & $91.300 €$ & 4 & $22.825 €$ & 87 & 22 & 87 \\
\hline Universidad Complutense de Madrid & 21 & 79 & $1.774 .464,05 €$ & 4 & $22.461,57 €$ & 2.104 & 27 & 100 \\
\hline Universidad de A Coruña & 1 & 0 & $27.805 €$ & 0 & No publicación & 0 & 0 & 0 \\
\hline Universidad de Alcalá & 2 & 4 & $74.882 €$ & 2 & $18.720,50 €$ & 14 & 4 & 7 \\
\hline Universidad de Almería & 1 & 2 & $37.000 €$ & 2 & $18.500 €$ & 9 & 5 & 9 \\
\hline Universidad de Cantabria & 2 & 3 & $131.775 €$ & 2 & $43.925 €$ & 133 & 44 & 67 \\
\hline Universidad de Deusto & 1 & 0 & $57.000 €$ & 0 & No publicación & 0 & 0 & 0 \\
\hline Universidad de Granada & 6 & 30 & $240.636,30 €$ & 5 & $8.021,21 €$ & 888 & 30 & 148 \\
\hline Universidad de Huelva & 1 & 3 & $22.769 €$ & 3 & $7.589,67 €$ & 2 & 1 & 2 \\
\hline Universidad de la Rioja & 1 & 0 & $73.916 €$ & 0 & No publicación & 0 & 0 & 0 \\
\hline Universidad de León & 3 & 4 & $143.886,22 €$ & 1 & $35.971,55 €$ & 5 & 1 & 2 \\
\hline Universidad de Lleida & 1 & 0 & $31.853,64 €$ & 0 & No publicación & 0 & 0 & 0 \\
\hline Universidad de Málaga & 2 & 0 & $71.949 €$ & 0 & No publicación & 0 & 0 & 0 \\
\hline Universidad de Murcia & 3 & 10 & $116.234,17 €$ & 3 & $11.623,42 €$ & 71 & 7 & 24 \\
\hline Universidad de Navarra & 3 & 18 & $208.683 €$ & 6 & $11.593,50 €$ & 401 & 22 & 134 \\
\hline Universidad de Oviedo & 6 & 37 & $287.914 €$ & 6 & $7.781,46 €$ & 441 & 12 & 74 \\
\hline Universidad de Salamanca & 6 & 9 & $191.330,97 €$ & 2 & $21.259 €$ & 126 & 14 & 21 \\
\hline Universidad de Santiago de Compostela & 13 & 51 & $904.235,99 €$ & 4 & $17.730,12 €$ & 850 & 17 & 65 \\
\hline Universidad de Sevilla & 4 & 16 & $221.422 €$ & 4 & $13.838,88 €$ & 485 & 30 & 121 \\
\hline Universidad de Valladolid & 1 & 1 & $26.444,53 €$ & 1 & $26.444,53 €$ & 0 & 0 & 0 \\
\hline Universidad del País Vasco & 3 & 13 & $97.871,35 €$ & 4 & $7.528,57 €$ & 225 & 17 & 75 \\
\hline Universidad Miguel Hernández & 6 & 28 & $321.616,52 €$ & 5 & $11.486,30 €$ & 742 & 27 & 124 \\
\hline $\begin{array}{l}\text { Universidad Nacional de Educación a } \\
\text { Distancia }\end{array}$ & 5 & 24 & $398.380,31 €$ & 5 & $16.599,18 €$ & 389 & 16 & 78 \\
\hline Universidad Rey Juan Carlos & 1 & 0 & $24.500 €$ & 0 & No publicación & 0 & 0 & 0 \\
\hline Universitat Autònoma de Barcelona & 3 & 29 & $301.892,38 €$ & 10 & $10.410,08 €$ & 552 & 19 & 184 \\
\hline Universitat de Barcelona & 10 & 50 & $639.986 €$ & 5 & $12.799,72 €$ & 915 & 18 & 92 \\
\hline Universitat de les Illes Balears & 5 & 19 & $256.437 €$ & 4 & $13.496,68 €$ & 238 & 13 & 48 \\
\hline Universitat de València & 12 & 60 & $554.979,27 €$ & 5 & $9.249,65 €$ & 707 & 12 & 59 \\
\hline Universitat Jaume I & 7 & 39 & $287.823,52 €$ & 6 & $7.380,09 €$ & 787 & 20 & 112 \\
\hline Universitat Pompeu Fabra & 7 & 63 & $549.636,85 €$ & 9 & $8.724,39 €$ & 1.171 & 19 & 167 \\
\hline Total & 253 & 1.050 & $16.051 .410,87 €$ & 4 & $15.287,06 €$ & 22.514 & & \\
\hline
\end{tabular}

Nota. NA: Número de Ayudas; NT: Número de trabajos; TA: Número de trabajos publicados por Ayuda; €/T: importe en euros por trabajo publicado. Un mismo artículo puede estar financiado por dos ayuda.

y la Fundación Pública Andaluza para la Investigación de Málaga en Biomedicina y Salud (FIMABIS) $(n=17)$. Por último, el indicador «importe por trabajo» $(€ / T)$ es de 261.197,46€ en la Fundación para la Investigación Biomédica del Hospital Universitario 12 de Octubre, pues de los cinco proyectos financiados sólo hubo una publicación derivada, y de $110.920 €$ en la Fundación para la Formación e Investigación Sanitaria de la Región de Murcia.
Respecto al número de citas, las instituciones de trabajo de los autores que más citas han generado han sido el Consejo Superior de Investigaciones Científicas, la Fundación de la Comunidad Valenciana Centro de Investigación Príncipe Felipe y la Universidad Complutense de Madrid con 2.591, 2.182 y 2.104 citas, respectivamente. La Fundación de la Comunidad Valenciana Centro de Investigación Príncipe Felipe se mantiene entre las tres principales insti- 
tuciones respecto al número de citas por trabajo $(\mathrm{n}=68)$ y número de citas por ayuda $(\mathrm{n}=727)$.

E1 99,7\% de los artículos derivados han sido realizados en colaboración. La media recortada al $5 \%$ del número de autores de los artículos derivados de los proyectos de financiación se sitúa en 6,5 autores por trabajo. La media de autores por trabajo es superior en ciencias de la salud $(7,15)$ que en ciencias básicas $(6,27)$ y que en ciencias sociales $(5,23)\left(\mathrm{K}-\mathrm{W}: \chi^{2}=40,61 ; \mathrm{p}<0.001\right)$. En el 17,2\% de los artículos no existe colaboración entre instituciones y en el $28,8 \%$ de los trabajos colabora una institución extranjera. La colaboración internacional es mayor en ciencias de la salud $(33,8 \%)$ respecto a las otras áreas $\left(\chi^{2}=14,3 ; \mathrm{p}<0,002\right)$. Se observan diferencias en el número de autores por trabajo según el tipo de colaboración (K-W: $\chi^{2}=174,2 ; \mathrm{p}<0,001$ ) (figura 1c). Los trabajos donde colaboran autores de varias instituciones nacionales e internacionales tienen un mayor número de autores por trabajo.

Los autores colaboraron con investigadores de 89 países. En la figura 3 se observa el mapa de colaboración entre países con un umbral de dos o más artículos en colaboración, con lo que se genera una red en la que participan 29 países. En ella se observa que el país con el que más colabora España es Estados Unidos $(n=112)$, seguido del Reino Unido $(\mathrm{n}=57)$ y Australia $(\mathrm{n}=43)$.

\section{Discusión}

Este trabajo ha permitido conocer e investigar aspectos de relevancia relacionados con la financiación de la investigación en trastornos adictivos en España por parte de la
Delegación del Gobierno para el Plan Nacional sobre Drogas y su rentabilidad académica en términos de publicaciones científicas y su impacto. El PNSD sufragó, por término medio, 15 ayudas anuales a proyectos de investigación, con un importe anual cercano al millón de euros. Casi tres cuartas partes de las ayudas tuvieron publicaciones derivadas, la gran mayoría de ellas en revistas extranjeras. La revista Adicciones fue la española en la que se publicaron un mayor número de artículos. Se identificó un alto grado de colaboración internacional y la mayoría de los artículos derivados son de investigación básica.

La financiación pública de la investigación en trastornos adictivos compite con otras prioridades de financiación de los gobiernos que tienen como objetivo mejorar la salud de la población. En España, la financiación de los proyectos de investigación en esta área proviene de la Unión Europea, el Gobierno de España, las Comunidades Autónomas, la industria farmacéutica, las fundaciones y las organizaciones no gubernamentales, al igual que en otras áreas de las ciencias de salud y de las ciencias sociales, y en el marco de lo que se ha venido a denominar modelo multinivel del fomento y promoción de la I+D+i (Fernández-Formoso, Pérez-Ortega, Sanz-Martiul y Blázquez-Herranz, 2010). Sin embargo, en el área de los trastornos adictivos el Gobierno de España es la principal fuente de financiación a través de las convocatorias del PNSD, desde el 2000, de la Acción Estratégica en Salud del Instituto de Salud Carlos III (s.f.) y del Plan Estatal de Investigación Científica y Técnica y de Innovación (Ministerio de Ciencia e Innovación, s.f.a, s.f.b), denominado anteriormente Plan Nacional I+D+I.

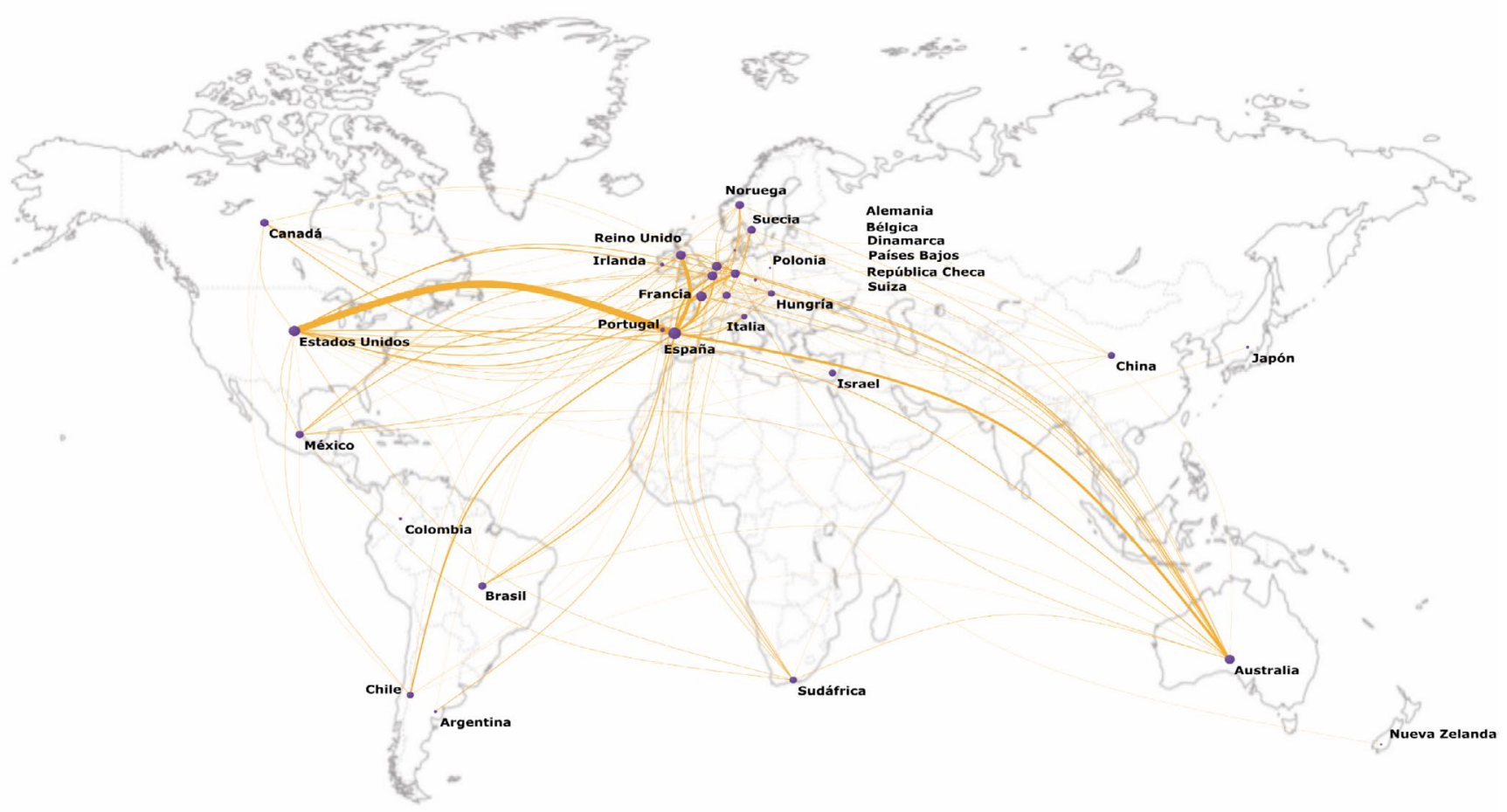

Figura 3. Red de colaboración de los países de los autores de los trabajos derivados. 
El análisis de las publicaciones derivadas de proyectos financiados ha sido objeto de estudio en algunas áreas biomédicas (Lewison y Dawson, 1998; Sun, Steinberg y Jagsi, 2013) como, por ejemplo, en nutrición (Thomson, 2007), genómica (Schiermeier, 1999), rehabilitación (DeLisa y Rosenthal, 2005; Zwingmann, Buschmann-Steinhage, Gerwinn y Klosterhuis, 2004), investigación sobre células madre (Campbell, 2005) y cardiología (Aleixandre et al., 2011; Rodríguez-Padial et al., 2019). Sin embargo, no hemos encontrado publicaciones que analicen el rendimiento en publicaciones de la inversión de organismos oficiales o asociaciones científicas y fundaciones en el área de los trastornos adictivos. La mayor parte de los estudios sobre financiación se refieren a la financiación de los tratamientos (Chalmers, Ritter, Berends y Lancaster, 2016; Mark, Levit, Vandivort-Warren, Coffey y Buck, 2007; Stewart y Horgan, 2011) y a la reducción de daños (Atun y Kazatchkine, 2010; Bridge et al., 2016; Kulesza, Teachman, Werntz, Gasser y Lindgren, 2015; World Health Organization, 2012).

En relación con las ayudas concedidas por el PNSD, nuestro análisis ha mostrado que los proyectos financiados son fundamentalmente del área de las ciencias básicas, área en la que se presenta un mayor número de proyectos y se le asigna una mayor dotación económica, mientras que el número de proyectos en ciencias sociales es menor y se les asigna también una cuantía inferior. Un estudio sobre las prácticas de financiación del Research Council of Norway's Programs for Mental Health encontró que los proyectos que financiaba estaban centrados fundamentalmente en aspectos biológicos, genéticos y neurológicos, mientras que eran menos frecuentes los dedicados a la salud comunitaria y a las condiciones de vida (Andersen, Borg, Karlsson y Larsen, 2016).

Como afirma Becoña (2016), la financiación del National Institute on Drug Abuse de Estados Unidos y de las instituciones españolas se ha centrado probablemente, por diversos factores, en la enfermedad cerebral y en la perspectiva biologicista, siendo necesaria más investigación en el área de prevención y otros modelos de actuación. Es posible que la estandarización metodológica que se utiliza en las ciencias básicas propicie que en la evaluación de los proyectos se produzca una mayor aprobación y financiación. Sería recomendable, por lo tanto, establecer factores de corrección en las convocatorias para dar más cabida a proyectos de otras áreas de conocimiento que también encuentran más dificultades en otras convocatorias nacionales. Por otra parte, cabe señalar que aun reconociendo la importancia de las subvenciones del PNSD, el porcentaje del producto interior bruto (PIB) dedicado a la investigación en España (1,21\% del PIB) está lejos de las aportaciones de los principales países europeos como Francia, que destina el 2,19\%, o Alemania, que destina el 3,04\%, e incluso China (2,13\%) y Estados Unidos (2,80\%) (Banco Mundial, 2019).
Los estudios previos que analizaron las publicaciones derivadas de proyectos de investigación en otras áreas han mostrado resultados dispares. Así, en dos estudios sobre el rendimiento de los proyectos cardiológicos financiados por la Sociedad Española de Cardiología en dos periodos de tiempo diferentes se encontraron porcentajes de publicación derivada en el 59,4\% de los proyectos en un caso (Aleixandre et al., 2011) y del 37\% en el otro (Rodríguez-Padial et al., 2019). En nuestro estudio, el 71,9\% de las ayudas han tenido publicaciones derivadas, porcentaje que se acerca bastante al primer estudio mencionado. No obstante, es probable que existan diferencias metodológicas que pueden explicar las discrepancias, según se cuenten solo los artículos derivados que reconocen la ayuda recibida en el manuscrito o se cuenten todos los trabajos, ya sea con el reconocimiento explícito de la ayuda concedida o sin él. Sin embargo, el hecho de que el porcentaje de publicaciones derivadas obtenido en nuestro estudio sea superior a un área tan consolidada como la cardiología muestra la importancia de la investigación que se realiza en trastornos adictivos. El número de publicaciones derivadas es mayor en el área de investigación básica, área que recibe más financiación por proyecto.

El mayor número de proyectos financiados es sobre alcohol, drogas en general y cocaína. Esto se relaciona con el hecho de que el alcohol es la droga más consumida, tanto en población general como adolescente, y con que las investigaciones en ciencias sociales suelen realizarse sobre todas las drogas, como ocurre, por ejemplo, en los estudios sobre substratos neurobiológicos y en los de prevención. El importante número de proyectos sobre cocaína se relaciona con el elevado número de artículos que publican los investigadores españoles sobre esta droga (Khalili et al., 2018). Actualmente, cuando se ha producido un incremento en el consumo de cannabis, la aparición de cannabinoides sintéticos y se ha abierto el debate internacional sobre el uso médico o recreativo del cannabis y consecuencias de su legalización (Cerdá et al., 2020; Matielo, et al., 2018; Smart y Pacula, 2019), sería aconsejable potenciar la investigación sobre esta droga, sobre todo tras observar un descenso del $58 \%$ en los proyectos financiados relacionados con el cannabis en la primera mitad del periodo estudiado (23 proyectos) respecto segundo periodo (11 proyectos).

Como se ha visto, las ayudas se han repartido entre grupos de investigación pertenecientes a una amplia variedad de instituciones beneficiarias que incluyen universidades, hospitales, institutos de investigación y fundaciones públicas y privadas de todo el estado. La rentabilidad en publicaciones derivadas de estas instituciones ha sido inconstante y debe tenerse en cuenta que no se han considerado otras variables que podrían relativizar esta rentabilidad, como el tamaño de los equipos de investigación o que un investigador puede solicitar la ayuda a través de diversas instituciones a las que puede estar adscrito. 
El hecho de que el $92 \%$ de las publicaciones derivadas se publicara en revistas extranjeras es una muestra de la internacionalización de la investigación española en trastornos adictivos, lo que confiere, además, una alta visibilidad. Por otra parte, casi el $80 \%$ de las revistas más productivas tienen unos factores de impacto que las sitúan en el primer o segundo cuartil en sus correspondientes áreas temáticas del Journal Citation Reports, lo que es una muestra de su calidad. Es importante resaltar que la internacionalización de la investigación está indisolublemente ligada a factores como las inversiones económicas y los recursos humanos destinados a la investigación (González, Valderrama y Aleixandre, 2012; Moya Anegón et al., 2008; Peters, 2006). Las áreas temáticas de las revistas dónde más se ha publicado son las de trastornos por abuso de sustancias, neurociencias y neurología, psiquiatría y farmacología, áreas en las que se suelen publicar numerosos artículos sobre los trastornos adictivos y su tratamiento (Tran et al., 2019). Sin embargo, se observa un déficit de publicaciones en revistas de las áreas de medicina general, salud pública y educación, áreas fundamentales en el abordaje de los trastornos adictivos. El hecho de que los artículos de la revista Drug and Alcohol Dependence hayan recibido el mayor número de citas ya había sido encontrado en un estudio previo sobre el mapa científico del tratamiento de las adicciones (Blobaum, 2013).

El número medio de autores por trabajo obtenido de 6,5 es superior al encontrado en el marco del estudio de 50 años de investigación en España en trastornos adictivos financiado por el Plan Nacional sobre Drogas (Aleixandre-Benavent et al., 2020d) y también es superior al encontrado en otro estudio sobre trastornos de la conducta alimentaria, donde la media se situaba en 4,39 autores por trabajo (Valderrama-Zurián et al., 2017). Las publicaciones derivadas de los proyectos investigación en ciencias de la salud presentan una media de número de autores por trabajo mayor que las derivadas de proyectos de ciencias básicas o ciencias sociales.

El estudio de colaboración en las publicaciones derivadas muestra que el $82,8 \%$ de los trabajos se realizaron en colaboración nacional o internacional y que esta colaboración se refleja en un mayor número de autores en los trabajos. En las publicaciones derivadas se colabora con 87 países y el principal país ha sido Estados Unidos. Estos datos ponen de manifiesto que la investigación española sobre drogodependencias se formaliza con investigadores de todos los continentes y que ha tenido un importante impacto la inclusión de la posibilidad de financiación de estancias formativas en Estados Unidos avaladas por el Instituto Nacional sobre Drogas de Abuso (NIDA). También debe resaltarse la posible influencia de la creación en el año 2002 de la Red de Trastornos Adictivos (RTA) en la iniciativa del Ministerio de Sanidad para impulsar la investigación en red (Rodríguez de Fonseca et al., 2006). Tanto el alto grado de colaboración entre autores como el elevado porcentaje de trabajos realizados en colaboración internacional en los artículos derivados concuerda con resultados previos en los que los artículos en el área de los trastornos adictivos que han sido financiados presentan una mayor media de autor por trabajo, un mayor grado de colaboración internacional y un mayor número de citas por artículo (Valderrama-Zurián, Castelló-Cogollos, Melero-Fuentes, Aleixandre-Benavent y Bueno-Cañigral, 2019).

\section{Limitaciones}

Este trabajo tiene algunas limitaciones que deben considerarse. Primero, es posible que algunas investigaciones pueden haberse pasado por alto si los investigadores principales de las ayudas no informaron de su publicación, o si estas se publicaron en revistas no indexadas en la Colección Principal de WoS o en Scopus. Sin embargo, las bases de datos consideradas en este trabajo se consideran hoy en día como el patrón de oro pues gozan de un prestigio internacional significativo y son las más utilizadas en los estudios bibliométricos, sobre todo los que analizan las métricas de citación e impacto científico (Moed, 2009). En segundo lugar, es posible que algunos autores hayan seguido una estrategia de fragmentación de publicaciones para alcanzar una mayor rentabilidad académica de sus proyectos, una práctica que solo sería posible identificar mediante una minuciosa revisión de las posibles publicaciones fragmentadas, aspecto que escapa del objetivo de nuestro estudio. También puede existir un «sesgo de financiación» por exceso o por defecto. El sesgo por exceso se debe a que algunos autores incluyen en su trabajo el agradecimiento a un proyecto financiado, independientemente del que el trabajo este realmente asociado a ese proyecto, con el objetivo de incrementar la productividad de los proyectos en las memorias, o que incluso sigan agradeciéndolo muchos años después de la financiación y sin tener relación aparente. Por el contrario, el «sesgo de financiación» por defecto está producido por la omisión del agradecimiento por parte de los autores en un trabajo financiado. En tercer lugar, es posible que al no disponer del investigador principal de los proyectos financiados en el periodo 2000-2003 se haya producido una infrarrepresentación de las publicaciones derivadas de estos proyectos. Por último, es evidente que los proyectos concedidos en los últimos años han tenido menos tiempo para publicar sus resultados, lo que puede haber producido un porcentaje menor de publicaciones derivadas y de sus citas.

A pesar de estas limitaciones, los resultados de nuestro análisis proporcionan información de interés para conocer el estado actual de la investigación en trastornos adictivos, los aspectos que pueden merecer más atención y aquellos en los que se encuentran las mayores dificultades. La información obtenida puede ser útil para tomar decisiones 
estratégicas destinadas a corregir las debilidades detectadas o a continuar financiando los aspectos más relevantes identificados.

\section{Conclusiones}

Los resultados de este estudio han permitido conocer el retorno de la inversión económica en proyectos de investigación del PNSD en términos de publicaciones científicas y pueden ayudar a determinar las futuras prioridades de financiación. La tasa de publicación obtenida, con la mayor parte de los trabajos publicados en revistas situadas en los primeros cuartiles del JCR de gran difusión e impacto internacional, indican que los equipos de investigación españoles realizan una investigación rigurosa y de calidad, que suelen colaborar en redes nacionales e internacionales y que suelen optimizar adecuadamente los recursos que obtienen. Además, este retorno supone un fuerte aliciente que debería persuadir al PNSD a persistir y a asegurar que se mantenga o que se amplíe la financiación de la investigación en trastornos adictivos.

Trabajos futuros podrían seguir la evolución de las ayudas del PNSD y de otras acciones públicas o privadas y de su rendimiento académico en términos de publicaciones, así como su impacto en la sociedad mediante la transferencia del conocimiento.

\section{Reconocimientos}

Delegación de Gobierno para el Plan Nacional sobre Drogas (convocatoria 2016. Proyecto 2016/028). Ministerio de Sanidad Consumo y Bienestar Social.

Servicio de Drogodependencias (PMD/UPCCA-València). Concejalía de Sanidad y Consumo. Ajuntament de València.

\section{Referencias}

Aleixandre Benavent, R. (1999). Investigación española en drogodependencias. ¿Hacia dónde vamos? Trastornos Adictivos, 1. 227-233.

Aleixandre-Benavent, R., Agulló-Calatayud, V., Alonso-Arroyo, A., Castelló-Cogollos, L., Lucas-Domínguez, R., Melero-Fuentes, D., ... Valderrama-Zurián, J. C. (2020a). Financed projects from Government Delegation grants for the National Plan on Drugs (Spain), 2000-2016. doi:10.5281/zenodo.3778579.

Aleixandre-Benavent, R., Agulló-Calatayud, V., Alonso-Arroyo, A., Castelló-Cogollos, L., Lucas-Domínguez, R., Melero-Fuentes, D., ... Valderrama-Zurián, J. C. (2020b). Financed researchs from Government Delegation grants for the National Plan on Drugs (Spain), 2001-2019. doi:10.5281/zenodo.3778642.

Aleixandre-Benavent, R., Agulló-Calatayud, V., Alonso-Arroyo, A., Castelló-Cogollos, L., Lucas-Domínguez,
R., Melero-Fuentes, D., ... Valderrama-Zurián, J. C. (2020c). Tables and figures of the supplementary material from research: Financed researchs from Government Delegation grants for the National Plan on Drugs: research assessment and scientific impact. doi: 10.5281/zenodo.4030455.

Aleixandre-Benavent, R., Agulló-Calatayud, V., Alonso-Arroyo, A., Castelló-Cogollos, L., Lucas-Domínguez, R., Melero-Fuentes, D., ... Valderrama-Zurián, J. C. (2020d). La evolución de la investigación española en drogodependencias presente en publicaciones cientificas. Recuperado de http://pnsd.uisys.es.

Aleixandre Benavent, R., Alonso Arroyo, A., Anguita Sánchez, M., Bolaños Pizarro, M., Heras, M., González Alcalde, G., ... Morell Cabedo, S. (2011). Evolución y repercusión científica de las becas de investigación de la Sociedad Española de Cardiología y la Fundación Española del Corazón (2000-2006). Revista Española de Cardiología, 64, 904-915. doi:10.1016/j.recesp.2011.03.024.

Álvarez, F. J., y del Río, M. C. (2003). Current initiatives in drug abuse research in Spain. Addiction, 98, 861. doi:10.1046/j.1360-0443.2003.04511.x.

Andersen, A. J. W., Borg, M., Karlsson, B. E. y Larsen, I. B. (2016). More of the same? A study of the funding practices of The Research Council of Norway's Programs for Mental Health in the period 1995 to 2015. Scandinavian Psychologist, 3. doi:10.15714/scandpsychol.3.e13.

Atun, R. y Kazatchkine, M. (2010). The Global Fund's leadership on harm reduction: 2002-2009. International Journal of Drug Policy, 21, 103-106. doi:10.1016/j.drugpo.2010.01.002.

Ballesteros, J., Torrens, M. y Valderrama Zurián, J.C. (coords.) (2006). Manual introductorio a la investigación en drogodependencias. Valencia: Sociedad Española de Toxicomanías.

Banco Mundial (2019). Gasto en investigación y desarrollo (\% del PIB). Recuperado de https://datos.bancomundial. org/indicador/GB.XPD.RSDV.GD.ZS.

Bar-Ilan, J., Levene, M. y Lin, A. (2007). Some measures for comparing citation databases. Journal of Informetrics, 1, 26-34. doi:10.1016/j.joi.2006.08.001.

Becoña, E. (2016). Adicción: no sólo un mal funcionamiento cerebral. Papeles del Psicólogo, 37, 118-125.

Blobaum, P. M. (2013). Mapping the literature of addictions treatment. Journal of the Medical Library Association, 101, 101-109. doi:10.3163/1536-5050.101.2.005.

Bornmann, L. y Leydesdorff, L. (2014). Scientometrics in a changing research landscape. EMBO Reports, 15, 12281232. doi:10.15252/embr.201439608.

Bridge, J., Hunter, B. M., Albers, E., Cook, C., Guarinieri, M., Lazarus, J. V., ... Wolfe, D. (2016). The Global Fund to Fight AIDS, Tuberculosis and Malaria's investments in harm reduction through the rounds-based funding model (2002-2014). International Journal of Drug Policy, 27, 132-137. doi:10.1016/j.drugpo.2015.08.001. 
Byrne, F. y Chapman S. (2005). The most cited authors and papers in tobacco control. Tobacco Control, 14, 155-160. doi:10.1136/tc.2005.011973.

Campbell, A. (2005). Ethos and economics: Examining the rationale underlying stem cell and cloning research policies in the United States, Germany, and Japan. American Journal of Law E Medicine, 31, 47-86. doi:10.1177/009885880503100102.

Cerdá, M., Mauro, C., Hamilton, A., Levy, N. S., Santaella-Tenorio, J., Hasin, D., ... Martins, S. S. (2020). Association Between Recreational Marijuana Legalization in the United States and Changes in Marijuana Use and Cannabis Use Disorder From 2008 to 2016. JAMA Psychiatry, 77, 165. doi:10.1001/jamapsychiatry.2019.3254.

Chalmers, J., Ritter, A., Berends, L. y Lancaster, K. (2015). Following the money: Mapping the sources and funding flows of alcohol and other drug treatment in Australia. Drug and Alcohol Review, 35, 255-262. doi:10.1111/ dar. 12337.

DeLisa, J. A. y Rosenthal, M. (2005). Funding for rehabilitation medicine. American Journal of Physical Medicine EF Rehabilitation, 84, 991-998. doi:10.1097/01. phm.0000187849.87038.33.

Fernández-Formoso, J. A., Pérez-Ortega, J. M., Sanz-Martiul, E. y Blázquez-Herranz, M. (2010). Para que nada ni nadie se quede sin investigar: la Acción Estratégica en Salud (AES) en el contexto del fomento y promoción de la I+D+i biosanitarias. Emergencias, 22, 460-469.

González Alcaide, G., Valderrama Zurián J. C. y Aleixandre Benavent, R. (2012). Análisis del proceso de internacionalización de la investigación científica española. Revista Española de Documentación Científica, 35, 94-118.

González-Alcaide, G., Calafat, A., Becoña, E., Thijs, B. y Glänzel, W. (2016). Co-Citation Analysis of Articles Published in Substance Abuse Journals: Intellectual Structure and Research Fields (2001-2012). Journal of Studies on Alcohol and Drugs, 77, 710-722. doi:10.15288/ jsad.2016.77.710.

Instituto de Salud Carlos III (s.f.). Acción Estratégica en Salud. Recuperado de https://www.isciii.es/QueHacemos/Financiacion/Paginas/Accion-Estrategica-en-Salud.aspx.

Khalili, M., Rahimi-Movaghar, A., Shadloo, B., Mojtabai, R., Mann, K. y Amin-Esmaeili, M. (2018). Global scientific production on illicit drug addiction: A two-decade analysis. European Addiction Research, 24, 60-70. doi:10.1159/000487590.

Kingwell B.A., Anderson G.P., Morris, M.E. Duckett S.J., Hoole, E.A., Rothwell-Short, J. y Wilson, A.J. (2006). Evaluation of NHMRC funded research completed in 1992, 1997 and 2003: gains in knowledge, health and wealth. Medical Journal of Australia, 184, 282286. doi:10.5694/j.1326-5377.2006.tb00238.x.

Kulesza, M., Teachman, B. A., Werntz, A. J., Gasser, M. L. y Lindgren, K. P. (2015). Correlates of public support toward federal funding for harm reduction strategies. Substance Abuse Treatment, Prevention, and Policy, 10. doi:10.1186/s13011-015-0022-5.

Lewison, G. y Dawson, G. (1998). The effect of funding on the outputs of biomedical research. Scientometrics, 41, 1727. doi:10.1007/bf02457963.

Ley $17 / 2003$, de 29 de mayo, por la que se regula el Fondo de bienes decomisados por tráfico ilícito de drogas y otros delitos relacionados, Boletín Oficial del Estado núm. 129 (2003). Recuperado de https://www.boe.es/ buscar/act.php?id = BOE-A-2003-10828.

Mahabee-Gittens, E. M., Gordon, J. S., Melink, K. F. y Merianos, A. L. (2017). Top 100 Cited Articles in Recent Tobacco Research. Journal of Behavioral Health, 6, 16-25.

Mark, T. L., Levit, K. R., Vandivort-Warren, R., Coffey, R. M. y Buck, J. A. (2007). Trends in spending for substance abuse treatment, 1986-2003. Health Affairs, 26, 11181128. doi:10.1377/hlthaff.26.4.1118.

Marx, W., Schier, H. y Wanitschek, M. (2001). Citation analysis using online databases: Feasibilities and shortcomings. Scientometrics, 52, 59-82. doi:10.1023/a:1012798911792.

Matielo, C., Sarzi, D., Justolin, B., Lemos, R., Camargo, F. y Stefenon, V. (2018). A bibliometric analysis of cannabis publications: Six decades of research and a gap on studies with the plant. Publications, 6, 40. doi:10.3390/ publications6040040.

Ministerio de Ciencia e Innovación (s.f.a). Plan Estatal de Investigación Científica y Técnica y de Innovación 20172020. Recuperado de http://www.ciencia.gob.es/ portal/site/MICINN/menuitem.7eeac5cd345b4f34f$09 \mathrm{dfd} 1001432 \mathrm{ea} 0 /$ ?vgnextoid $=83 \mathrm{~b} 192 \mathrm{~b} 9036 \mathrm{c} 2210 \mathrm{Vgn}-$ VCM1000001d04140aRCRD.

Ministerio de Ciencia e Innovación (s.f.b). Planes Nacionales $I+D+i$ Anteriores. Recuperado de http://www.ciencia. gob.es/portal/site/MICINN/menuitem.29451c2ac$1391 \mathrm{f} 1 \mathrm{febebed} 1001432 \mathrm{ea} 0 /$ ?vgnextoid $=79 \mathrm{dbec}-$ 05f2a7d210VgnVCM1000001d04140aRCRD.

Ministerio de Sanidad, Servicios Sociales e Igualdad (2019). Ayudas para la realización de programas supracomunitarios sobre adicciones en el año 2019. Recuperado de https://www.ayudas-subvenciones.es/subvencion/17851/ ayudas-para-la-realizacion-de-programas-supracomunitarios-sobre-adicciones-en-el-ano-2019.

Moed, H. F. (2009). New developments in the use of citation analysis in research evaluation. Archivum Immunologiae et Therapiae Experimentalis, 57, 13-18. doi:10.1007/ s00005-009-0001-5.

Moya Anegón, F. de, Chinchilla Rodríguez, Z., Benavent Pérez, M., Corera-Álvarez, E., González Molina, A. y Vargas Quesada, B. (2008). Indicadores bibliométricos de la actividad científica española 2008. Madrid: Fundación Española para la Ciencia y la Tecnología (FECYT).

Peters, M. A. (2006). The rise of global Science and the emerging political economy of international research 
collaborations. European Journal of Education, 41, 225244.

Plan Nacional sobre Drogas (s.f.a). Convocatoria de ayudas para el desarrollo de proyectos de investigación sobre adicciones. Recuperado de http://www.pnsd.mscbs.gob.es/delegacionGobiernoPNSD/convocatoriaSubvenciones/ongs/ proyecInvestig.htm.

Plan Nacional sobre Drogas (s.f.b). Programas de Corporaciones Locales. Recuperado de http:/ / www.pnsd.mscbs.gob. es/delegacionGobiernoPNSD/convocatoriaSubvenciones/ongs/programasCorpora.htm.

Real Decreto 1677/1985, de 11 de septiembre, de Coordinación Interministerial para la ejecución del Plan Nacional sobre Drogas, Boletín Oficial del Estado núm. 226. (1985). Recuperado de https://www.boe.es/buscar/doc.php?id=BOE-A-1985-19702.

Rodríguez de Fonseca, F., Aleixandre, R., Camí, J., Navarro, M., Torrens, M., Iraurgi, J., ... Molina, M. (2006). La investigación en drogodependencias. Trastornos Adictivos, 8, 115-132.

Rodríguez-Padial, L., Fernández Lozano, I., Hidalgo Urbano, R., Silva Melchor, L., Evangelista Massip, A., Anguita Sánchez, M. y Íñiguez Romo, A. (2019). Evolución e impacto bibliométrico de las becas de la Sociedad Española de Cardiología/Fundación Española del Corazón en el periodo 2007-2012. Revista Española de Cardiología, 72, 1012-1019. doi:10.1016/j.recesp.2018.08.013.

Schiermeier, Q. (1999). Berlin places genomics among top funding priorities. Nature, 402, 568-568. doi: $10.1038 / 45036$.

Smart, R. y Pacula, R. L. (2019). Early evidence of the impact of cannabis legalization on cannabis use, cannabis use disorder, and the use of other substances: Findings from state policy evaluations. The American Journal of Drug and Alcohol Abuse, 45, 644-663. doi:10.1080/009529 90.2019.1669626.

Stewart, M. T. y Horgan, C. M. (2011). Health services and financing of treatment. Alcohol Research $\xi^{2}$ Health, 33, 389-394

Sun, G. H., Steinberg, J. D. y Jagsi, R. (2013). Funding for Biomedical Research. JAMA, 309, 1228-1229.

Tanner-Smith, E. E. y Polanin, J. R. (2016). Brief alcohol intervention trials conducted by higher prestige authors and published in higher impact factor journals are cited more frequently. Journal of Clinical Epidemiology, 75, 119125. doi:10.1016/j.jclinepi.2016.01.028.

Thomson, C. A. (2007). Funding Nutrition Research: Where's the Money? Nutrition in Clinical Practice, 22, 609-617. doi:10.1177/0115426507022006609.

Tran, B. X., Hoang, C. L., Tam, W., Phan, H. T., Vu, G. T., Latkin, C., ... Ho, R. C. M. (2019). A global bibliometric analysis of antiretroviral treatment adherence: implications for interventions and research development (GA-
PRESEARCH). AIDS Care, 32, 637-644. doi:10.1080/095 40121.2019.1679708.

Valderrama-Zurián, J. C., Castelló-Cogollos, L., Melero-Fuentes, D., Aleixandre-Benavent, R. y Bueno-Cañigral, F. J. (2019). Bibliometric differences between funding and non-funding papers on substance abuse scientific research. En: G. Catalano, C. Daraio, M. Gregori, H. F. Moed y G. Ruocco (Eds.), 17th International Conference on Scientometrics and Informetrics (pp. 26222623). Roma: Edizioni Efesto.

Valderrama-Zurián, J.-C., Aguilar-Moya, R., Cepeda-Benito, A., Melero-Fuentes, D., Navarro-Moreno, M.-Á., Gandía-Balaguer, A. y Aleixandre-Benavent, R. (2017). Productivity trends and collaboration patterns: A diachronic study in the eating disorders field. PLoS One, 12, e0182760. doi:10.1371/journal.pone.0182760.

van Wesel, M. (2015). Evaluation by Citation: Trends in Publication Behavior, Evaluation Criteria, and the Strive for High Impact Publications. Science and Engineering Ethics, 22, 199-225. doi:10.1007/s11948-015-9638-0.

Waltman, L. (2016). A review of the literature on citation impact indicators. Journal of Informetrics, 10, 365-391. doi:10.1016/j.joi.2016.02.007.

World Health Organization (2012). WHO, UNODC, UNAIDS technical guide for countries to set targets for universal access to HIV prevention, treatment and care for injecting drug users 2012 revision. Recuperado de https:/ / apps.who.int/iris/ bitstream/handle/10665/77969/9789241504379_eng. pdf;jsessionid $=$ 707AED61E667AFF1BCE59B4E5F2CDD13? sequence $=1$.

Zwingmann, C., Buschmann-Steinhage, R., Gerwinn, H. y Klosterhuis, H. (2004). Förderschwerpunkt «Rehabilitationswissenschaften»: Ergebnisse - Umsetzung - Erfolge und Perspektiven. Die Rehabilitation, 43, 260-270. doi:10.1055/s-2004-828393. 
